\title{
LIKELIHOOD OF RETROGRADE DOUBLE-J STENTING ACCORDING TO URETERAL OBSTRUCTING PATHOLOGY
}

\author{
ALEXANDRE DANILOVIC, IOANNIS M. ANTONOPOULOS, JOSE L. MESQUITA, \\ ANTONIO M. LUCON \\ Division of Urology, General Hospital, University of Sao Paulo Medical School, USP, Sao Paulo, Brazil
}

\begin{abstract}
Objectives: To evaluate the likelihood of retrograde double-J stenting in urgent ureteral drainage according to obstructing pathology.

Materials and Methods: From July 2002 to January 2003, 43 consecutive patients with ureteral obstruction who needed urgent decompression were evaluated at our institution, where we performed a total of 47 procedures. Emergency was defined as ureteral obstruction associated with infection, obstructive acute renal failure, or refractory pain. Ureteral obstruction was defined as intrinsic and extrinsic based on etiology and evaluated by ultrasound. Patients submitted to previous double-J stenting were excluded. Failures in retrograde ureteral stenting were treated with percutaneous nephrostomy. Results were analyzed with Fisher's exact test and regression analysis.

Results: Failure in retrograde ureteral stenting occurred in 9\% (2/22) and 52\% (13/25) of the attempts in patients with intrinsic and extrinsic obstruction respectively $(\mathrm{p}<0.001)$. Failures in stenting extrinsic obstructions occurred due to lack of identification of the ureteral meatus in $77 \%$ and impossibility of catheter progression in $23 \%(\mathrm{p}<0.05)$. All attempts of retrograde catheter insertion failed in obstructions caused by prostate or bladder pathologies (6/6). Inability to identify the ureteral meatus was the cause of all failures.

Conclusion: Retrograde double-J stenting has a low probability of success in extrinsic ureteral obstruction caused by prostate or bladder disease. Such cases might be best managed with percutaneous nephrostomy.
\end{abstract}

Key words: ureter; obstruction; drainage; stents

Int Braz J Urol. 2005; 31: 431-6

\section{INTRODUCTION}

Ureteral obstruction often presents as urological urgency demanding surgical treatment with urinary diversion (1-5). The first successful endoscopic ureteral drainage using a silicone catheter was reported by Zimskind et al. in 1967 (6). During the last decade, double-J stenting has been widely used by urologists. Despite endourological technical advances, retrograde double-J stenting may be cum- bersome or impossible. Alternatively, one may prefer percutaneous nephrostomy, an efficient method but with the inconvenience of being an external diversion $(1-5,7,8)$. The choice of double-J stenting or percutaneous nephrostomy for urgent ureteral decompression is controversial and oriented by surgeon preference (4). There are a few studies on this issue and most of them are retrospective involving elective procedures $(1,2,5,7-9)$. We conducted a prospective study to evaluate the success of retrograde 
double-J stenting in urgent ureteral drainage and to define criteria for selection of decompression method in order to reduce cost and to avoid time loss.

\section{MATERIALS AND METHODS}

Between July 2002 and January 2003, 43 consecutive patients with ureteral obstruction and need of urgent decompression were evaluated at our institution, where we performed a total of 47 procedures. The need for urgent decompression was defined as ureteral obstruction associated with infection, obstructive acute renal failure, or refractory pain. All patients were evaluated with x-ray (KUB) and ultrasound in order to diagnose obstructive uropathy (10). Non-enhanced spiral CT was performed when standard evaluation was not satisfactory. Patients submitted to previous retrograde double-J stenting were excluded.

Ureteral obstruction was classified accordingly to etiology as intrinsic (inside the ureteral lumen) or extrinsic (outside the ureteral lumen) $(1,9,11,12)$.

All procedures were performed under general anesthesia, with fluoroscopic C-arm guidance (13). Retrograde pyelography was performed previously to each procedure when it was possible to identify the ureteral meatus. This was done using an openended ureteral catheter. This catheter was then used to pass a $0.35 \mathrm{~mm}$ hydrophilic guide wire (13). A nonhydrophilic polyurethane double-J ureteral catheter of various sizes $(4,7 ; 6 ; 7 \mathrm{Fr})$ was used according to surgeon's preference $(14,15)$.

The adequate positioning of the double-J stent was confirmed by fluoroscopy at the end of the procedure. Failures in retrograde ureteral stenting were immediately treated with percutaneous nephrostomy. The percutaneous nephrostomy kit used was $14 \mathrm{~F} / 4.6 \mathrm{~mm}$. The success of percutaneous nephrostomy placement was confirmed with antegrade pyelography after the procedure.

Statistical analysis was performed with Fisher's exact test and regression analysis, with $\mathrm{p}<$ 0.05 considered significant.

\section{RESULTS}

Intrinsic and extrinsic lesions were responsible for $47 \%$ and $53 \%$ of the obstructions respectively (Table-1).

Intrinsic (Table-2) and extrinsic (Table-3) groups were sex and age matched (Table-4).

The need for ureteral decompression differed between groups. The main indication for decompression in the intrinsic group was pyelonephritis (77\%) and in the extrinsic group it was acute renal failure $(88 \%)$. The site of obstruction was preferentially distal in extrinsic lesions, and proximal in intrinsic ones ( $84 \%$ vs. $41 \%, \mathrm{p}<0.001)$, and renal dilation was more pronounced in the extrinsic group (27\% vs. $44 \%, \mathrm{p}<$ $0.05)$.

The results show that retrograde ureteral stenting success was significantly lower in patients with extrinsic ureteral obstruction (Table-5).

Retrograde ureteral stenting failures in intrinsic obstruction were caused by non-progression of the hydrophilic guide wire and by non-identification of the ureteral meatus (one case each). Failures in extrinsic obstruction were caused by non-progression of the hydrophilic guide wire in 3 patients (23\%) and by non-identification of the ureteral meatus in $10 \mathrm{pa}-$ tients $(77 \%)(\mathrm{p}<0.05)$.

Table 1 - Specific causes of ureteral obstruction in 47 kidneys.

\begin{tabular}{lc}
\hline & N Kidneys (\%) \\
\hline Intrinsic & $\mathbf{2 2}(\mathbf{4 7})$ \\
Stone disease & 21 \\
Ureteral tumor & 1 \\
Extrinsic & $\mathbf{2 5}(\mathbf{5 3})$ \\
BPH & 1 \\
Colorectal carcinoma & 5 \\
Ovarian carcinoma & 2 \\
Uterine carcinoma & 8 \\
Prostate cancer & 4 \\
Endometrial carcinoma & 1 \\
Pancreas carcinoma & 1 \\
Testis cancer & 1 \\
Bladder cancer & 1 \\
Lymphoma & 1 \\
\hline
\end{tabular}


Table 2 - General data of the patients with intrinsic ureteral obstruction.

\begin{tabular}{|c|c|c|c|c|c|c|c|c|}
\hline Case & Sex & Age & Etiology & Side & Indication & Dilation & Site & Success \\
\hline 1 & Male & $53 y$ & Stone & Right & Pyelonephritis & Moderate & Distal & Yes \\
\hline 2 & Male & $10 \mathrm{y}$ & Stone & Left & Pain & Mild & Proximal & Yes \\
\hline 3 & Female & $46 y$ & Stone & Left & Pyelonephritis & Severe & Proximal & Yes \\
\hline 4 & Male & $46 y$ & Stone & Right & Pyelonephritis & Severe & Proximal & Yes \\
\hline 5 & Female & $64 y$ & Stone & Left & Pyelonephritis & Mild & Proximal & Yes \\
\hline 6 & Female & $42 y$ & Stone & Right & Pain & Mild & Distal & Yes \\
\hline 7 & Female & $67 y$ & Stone & Right & Pyelonephritis & Mild & Distal & Yes \\
\hline 8 & Male & $39 y$ & Stone & Right & Pyelonephritis & Mild & Distal & Yes \\
\hline 9 & Female & $42 y$ & Stone & Left & Pain & Mild & Medium & Yes \\
\hline 10 & Female & $22 y$ & Stone & Right & Pyelonephritis & Mild & Distal & Yes \\
\hline 11 & Male & $52 y$ & Stone & Right & Pyelonephritis & Mild & Medium & Yes \\
\hline 12 & Female & $21 \mathrm{y}$ & Stone & Right & Pyelonephritis & Moderate & Medium & Yes \\
\hline 13 & Female & $48 y$ & Stone & Right & Acute Renal Failure & Severe & Medium & No \\
\hline 14 & Male & $69 y$ & Stone & Left & Pyelonephritis & Severe & Proximal & Yes \\
\hline 15 & Female & $49 y$ & Stone & Right & Pyelonephritis & Mild & Distal & Yes \\
\hline 16 & Female & $48 y$ & Stone & Right & Pyelonephritis & Moderate & Proximal & Yes \\
\hline 17 & Female & $79 y$ & Stone & Left & Pyelonephritis & Moderate & Proximal & Yes \\
\hline 18 & Male & $47 y$ & Stone & Left & Pyelonephritis & Severe & Distal & Yes \\
\hline 19 & Female & $40 \mathrm{y}$ & Stone & Left & Pyelonephritis & Mild & Distal & Yes \\
\hline 20 & Female & $43 y$ & Stone & Left & Pyelonephritis & Moderate & Proximal & Yes \\
\hline 21 & Male & $63 y$ & Stone & Left & Pyelonephritis & Moderate & Distal & No \\
\hline 22 & Female & $56 y$ & Ureteral Tumor & Right & Acute Renal Failure & Severe & Proximal & Yes \\
\hline
\end{tabular}

All attempts of catheter insertion failed in obstructions caused by prostate or bladder pathologies (Table-6). Inability to identify the ureteral meatus was the cause of all failures.

One retrograde double-J insertion became complicated with ureteral perforation distally to the extrinsic obstruction and was managed with percutaneous nephrostomy. Follow-up was uneventful.

\section{COMMENTS}

The cornerstone for acute ureteral obstruction treatment is ureteral decompression. The ideal method should be minimally invasive, fast, and inexpensive. Currently, the most common methods in these situations are insertion of double-J catheter or placement of percutaneous nephrostomy. There is no consensus in the literature about which one is more appropriate, and usually the choice is left to the surgeon's preference (4). We evaluate the urgent ureteral decompression in patients with ureteral obstruction due to intrinsic and extrinsic pathologies. Retrograde double-J stenting failed in $9 \%(2 / 22)$ of intrinsic obstruction and in 52\% (13/25) of extrinsic obstruction $(\mathrm{p}<0.001)$. Yossepowitch and coworkers had a similar success index in patients with intrinsic ureteral obstruction and higher success index in selected cases of extrinsic obstruction (1). When the ureteral meatus was identified, retrograde pyelography was performed previously to each procedure. It did not alter the previous diagnosis of intra- or extra-ureteral obstruction, nevertheless it was useful to the detection of unexpected ureteral kinking. Failures of retrograde catheter insertion in extrinsic obstruction occurred due to non-identification of the ureteral meatus in $77 \%$ of the cases. Identification of ureteral meatus in patients with lower urinary tract conditions such as prostate and bladder pathologies was not possible in $100 \%$ 
Table 3 - General data of the patients with extrinsic ureteral obstruction.

\begin{tabular}{|c|c|c|c|c|c|c|c|}
\hline Case & Sex & Age & Etiology & Indication & Dilation & Site & Success \\
\hline 1 & Male & $84 y$ & $\mathrm{BPH}$ & ARF & Severe & Distal & No \\
\hline 2 & Male & $66 y$ & Prostate Tumor & ARF & Severe & Distal & No \\
\hline 3 & Male & $71 y$ & Prostate Tumor & ARF & Moderate & Distal & No \\
\hline 4 & Male & $56 y$ & Bladder Tumor & ARF & Severe & Distal & No \\
\hline 5 & Female & $30 y$ & Uterine Tumor & ARF & Moderate & Distal & No \\
\hline 6 & Female & $49 y$ & Lymphoma & ARF & Moderate & Distal & No \\
\hline 7 & Male & $75 y$ & Prostate Tumor & ARF & Severe & Distal & No \\
\hline 8 & Male & $75 y$ & Prostate Tumor & ARF & Moderate & Distal & No \\
\hline 9 & Male & $67 y$ & Colorectal Tumor & ARF & Moderate & Distal & No \\
\hline 10 & Male & $34 y$ & Testis Tumor & ARF & Severe & Distal & No \\
\hline 11 & Female & $78 y$ & Uterine Tumor & ARF & Severe & Distal & Yes \\
\hline 12 & Female & $78 y$ & Uterine Tumor & ARF & Severe & Distal & Yes \\
\hline 13 & Female & $41 y$ & Uterine Tumor & ARF & Moderate & Distal & Yes \\
\hline 14 & Female & $30 y$ & Uterine Tumor & ARF & Moderate & Distal & Yes \\
\hline 15 & Female & $57 y$ & Uterine Tumor & ARF & Severe & Distal & Yes \\
\hline 16 & Female & $57 y$ & Uterine Tumor & $\mathrm{ARF}$ & Moderate & Distal & Yes \\
\hline 17 & Female & $25 y$ & Ovarian Tumor & ARF & Mild & Distal & Yes \\
\hline 18 & Female & $52 y$ & Ovarian Tumor & ARF & Mild & Distal & Yes \\
\hline 19 & Male & $67 y$ & Colorectal Tumor & $\mathrm{ARF}$ & Moderate & Distal & Yes \\
\hline 20 & Female & $36 y$ & Uterine Tumor & Pyelonephritis & Severe & Distal & No \\
\hline 21 & Female & $46 y$ & Colorectal Tumor & ARF & Severe & Distal & No \\
\hline 22 & Female & $67 y$ & Pancreas Tumor & $\mathrm{ARF}$ & Mild & Medium & Yes \\
\hline 23 & Female & $64 y$ & Colorectal Tumor & Pyelonephritis & Moderate & Medium & Yes \\
\hline 24 & Female & $75 y$ & Endometrial Tumor & Pyelonephritis & Moderate & Distal & Yes \\
\hline 25 & Female & $35 y$ & Colorectal Tumor & ARF & Severe & Distal & No \\
\hline
\end{tabular}

$A R F=$ acute renal failure, $B P H=$ benign prostate hyperplasia

Table 4 - Demographic data of intrinsic and extrinsic obstruction cases.

\begin{tabular}{llcc}
\hline Sex & Intrinsic & Extrinsic & P value \\
\hline Male & 8 & 8 & 0.852 \\
Female & 14 & 13 & \\
Mean age (range) & $47.5(10-79)$ & $55(30-84)$ & 0.119 \\
\hline
\end{tabular}

Table 5 - Success index of double-J insertion between groups.

\begin{tabular}{lccc}
\hline & Intrinsic (\%) & Extrinsic (\%) & P value \\
\hline Success & $20(81)$ & $12(48)$ & $<0.001$ \\
Failure & $2(9)$ & $13(52)$ & \\
\hline
\end{tabular}

Table 6- Success index of double-J insertion versus type of disease causing extrinsic ureteral obstruction.

\begin{tabular}{lcrll}
\hline & $\begin{array}{c}\text { Prostate } \\
\text { and Bladder (\%) }\end{array}$ & $\begin{array}{c}\text { Other } \\
\text { Tumors }(\%)\end{array}$ & P value \\
\hline Success & 0 & $12(63.2)$ & $<0.05$ \\
Failure & $6(100)$ & $7(36.8)$ & \\
\hline
\end{tabular}

of cases. Therefore, attempts of retrograde catheter insertion in patients with lower urinary tract conditions may be avoided, giving preference to percutaneous nephrostomy.

Pearle and associates concluded that double$\mathrm{J}$ catheter and percutaneous nephrostomy are equally good methods for ureteral decompression in obstruc- 
tive ureterolithiasis associated with infection (7). However, double-J catheters are prone to obstruct when used for long periods. Docimo \& DeWolf reported a 30-day re-obstruction index up to $53 \%$ in extrinsic ureteral obstruction (9). Such problem may be adequately dealt with by simultaneous insertion of 2 double-J catheters in the obstructed ureteral unit $(4,11,12)$.

The impact in quality of life caused by temporary urinary diversion was accessed by Joshi \& colleagues and no functional or psychosocial difference between double-J catheter and percutaneous nephrostomy in ureteral decompression was found (5). Nevertheless, patients were followed for only 30 days. Possibly a longer follow-up may disclose differences between both methods. Our impression is that an external prosthesis promotes progressive loss of quality of life caused by more hospital visits due to nephrostomy displacement or infection.

The choice of ureteral drainage method should take cost into account. Both procedures are expensive as they are performed in the operating room under fluoroscopy. The double-J catheter used in the present study costs US\$ 47 and the percutaneous nephrostomy kit costs US\$ 88 . As the double-J catheter ensures adequate ureteral drainage, similar impact in quality of life and lower cost, it should be considered the preferential method for ureteral decompression except for selected cases.

\section{CONCLUSIONS}

Retrograde double-J stenting has a low probability of success in extrinsic ureteral obstruction caused by prostate or bladder disease. Such cases might be best managed with percutaneous nephrostomy.

\section{REFERENCES}

1. Yossepowitch O, Lifshitz DA, Dekel Y, Gross M, Keidar DM, Neuman M, et al.: Predicting the success of retrograde stenting for managing ureteral obstruction. J Urol. 2001; 166: 1746-9.
2. Mokhmalji H, Braun PM, Portillo FJ, Siegsmund M, Alken P, Kohrmann KU: Percutaneous nephrostomy versus ureteral stents for diversion of hydronephrosis caused by stones: a prospective, randomized clinical trial. J Urol. 2000; 165: 1088-92.

3. Pappas P, Stravodimos KG, Mitropoulos D, Kontonopoulou C, Haramoglis S, Giannopoulou M, et al.: Role of percutaneous urinary diversion in malignant and benign obstructive uropathy. J Endourol. 2000; 14: 401-5.

4. Watterson JD, Cadieux P, Denstedt JD: Ureteral stents: which, when, and why? AUA update series. 2002; vol. XXI, lesson 16, p. 122.

5. Joshi HB, Adams S, Obadeyi OO, Rao PN: Nephrostomy tube or "JJ" ureteric stent in ureteric obstruction: assessment of patient perspectives using quality-of-life survey and utility analysis. Eur Urol. 2001; 39: 695-701.

6. Zimskind PD, Kelter TR, Wilkerson SL: Clinical use of long-term indwelling silicone rubber ureteral splints inserted cystoscopically. J Urol. 1967; 97: 8404.

7. Pearle MS, Pierce HL, Miller GL, Summa JA, Mutz JM, Petty BA, et al.: Optimal meted of urgent decompression of the collecting system for obstruction and infection due to ureteral calculi. J Urol. 1998; 160: 1260-4.

8. Park DS, Park JH, Lee YT: Percutaneous nephrostomy versus indwelling ureteral stents in patients with bilateral non-genitourinary malignant extrinsic obstruction. J Endourol. 2002; 16: 153-4.

9. Docimo SG, DeWolf WC: High failure rate of indwelling ureteral stents in patients with extrinsic obstruction: experience at 2 institutions. J Urol. 1989; 142: 277-9.

10. Fernbach SK, Maizels M, Conway JJ: Ultrasound grading of hydronephrosis: introduction to the system used by the Society for Fetal Urology. Pediatr Radiol. 1993; 23: 478-80.

11. Rotariu P, Yohannes P, Alexianu M, Rosner D, Lee BR, Lucan M, et al.: Management of malignant extrinsic compression of the ureter by simultaneous placement of two ipsilateral ureteral stents. J Endourol. 2001; 15: 979-83.

12. Liu JS, Hrebinko RL: The use of 2 ipsilateral ureteral stents for relief of ureteral obstruction from extrinsic compression. J Urol. 1998; 159: 179-81.

13. Mata JA, Culkin DJ, Venable DD: Techniques for bypassing and stenting ureteral obstructions. J Urol. 1994; 152: 917-9. 
14. Candela JV, Bellman GC: Ureteral stents: impact of diameter and composition on patient symptoms. $\mathrm{J}$ Endourol. 1997; 11: 45-7.
15. Hübner WA, Plas EG, Stoller ML: The double-J ureteral stent: in vivo and in vitro flow studies. J Urol. 1992; 148: 278-80.

Received: February 24, 2005 Accepted after revision: June 28, 2005

\section{Correspondence address:}

Dr. Alexandre Danilovic

Rua Alves Guimarães, 623 / 161

05419-001, São Paulo, SP, Brazil

E-mail: alexandre.danilovic@sbu.org.br

\section{EDITORIAL COMMENT}

Double-J stenting has become an important endourological procedure in ureteral obstructive pathology. Successful stenting would reduce the morbidity of extrinsic ureteral obstruction. The authors reported a low success rate, especially in lower ureteral obstruction due to bladder or prostate pathology. Deployment of metallic ureteral stents would be a solution for overcoming the obstruction in this situation. Success would depend upon passing a guide wire. Failures in the retrograde approach can be overcome by antegrade stenting under ultrasound guided and fluoroscopic control. The upper tracts are usually dilated and easy to puncture. The guide wire can be negotiated into the bladder by using an angiogra- phy curved tip catheter. Once the guide wire is in the bladder it can be pulled outside the urethra by cystoscopy. By pulling the guide wire in the opposite direction the curvatures can be straightened out, making it easy to dilate over which one can put either double-J stent or metallic stent.

In my experience, combining an antegrade and retrograde approach to ureteral obstruction success can be increased remarkably.

\section{REFERENCE}

1. Kulkarni R: Metallic ureteric stents: the current situation. BJU Int. 2003; 92: 188-9.

Dr. Mahesh R. Desai Chair, Department of Urology Muljibhai Patel Urological Hospital Nadiad, Gujarat, India E-mail:mrdesai@mpuh.org 